\title{
Fellatio among male sanctuary-living chimpanzees during a period of social tension
}

\author{
Jake S. Brooker ${ }^{\mathrm{a}}$, Christine E. Webb ${ }^{\mathrm{b}}$ and Zanna Clay ${ }^{\mathrm{a}, *}$ \\ a Department of Psychology, Durham University, South Road, Durham DH1 3LE, UK \\ ${ }^{b}$ Department of Human Evolutionary Biology, Harvard University, 11 Divinity Avenue, \\ Cambridge, MA 02138, USA \\ *Corresponding author's e-mail address: zanna.e.clay@durham.ac.uk
}

Received 29 June 2020; initial decision 28 July 2020; revised 5 November 2020; accepted 30 November 2020; published online 15 December 2020

\begin{abstract}
Same-sex sexual behaviour has been documented across the animal kingdom, and is thought to reflect and enhance dyadic cooperation and tolerance. For instance, same-sex fellatio - the reception of a partner's penis into another's mouth — has been reported in several mammalian species other than humans. Although same-sex sexual behaviour is observed in our close relatives, the chimpanzees, fellatio appears to be very rare - as yet there are no published reports clearly documenting its occurrence. At Chimfunshi Wildlife Orphanage in Zambia, we observed an instance of fellatio occurring during a post-conflict period between two adult male chimpanzees (born and mother-reared at the sanctuary) where one of the males was the victim. We discuss this event with respect to the putative functions of homosexual behaviour in great apes. Given its rarity in chimpanzees, this fellatio between adult males also highlights the apparent behavioural flexibility present in our close relatives.
\end{abstract}

\section{Keywords}

chimpanzee, fellatio, same-sex sexual behaviour, homosexual behaviour, sanctuary, behavioural flexibility, social tension.

\section{Introduction}

Same-sex sexual interactions, i.e., genital contacts, mounting or other sexual solicitations between same-sex partners have been documented in many animal taxa including mammals, birds, marine life, and insects (Sommer \& 
Vasey, 2006; Bailey \& Zuk, 2009). A varied and widespread behaviour in the natural world, homosexual or same-sex sexual behaviour may have diverse as well as species-specific functions (Hanby, 1976; Moscovice et al., 2019), and, in some cases, represent idiosyncratic innovations for behaviourally flexible animals. Among our close relatives, the nonhuman primates, proposed functions include promoting cooperation and coalitionary support as well as preventing and reducing social tension, such as through post-conflict reconciliation (Vasey, 1996; Paoli et al., 2007; Clay \& de Waal, 2013; Moscovice et al., 2019). Nevertheless, as has been suggested for same-sex mounting among female Japanese macaques (Vasey, 2006), same-sex sexual behaviours do not always appear to convey an adaptive benefit.

Although same-sex sexual behaviour is habitual and particularly pronounced among bonobos (de Waal, 1990), chimpanzees (Pan troglodytes) are also know to engage in same-sex sexual behaviour across multiple contexts (Savage-Rumbaugh \& Wilkerson, 1978). Pan same-sex sexual behaviours include mounting, genital touching, and rump-rump touching (de Waal, 1988; Goodall, 1989). Although same-sex sexual behaviours can occur in a range of contexts in animal taxa, in both chimpanzees and bonobos, these behaviours are especially common during socially tense periods including pre-feeding, post-conflict periods, inter-group encounters, and, for chimpanzees, before boundary patrols (Paoli et al., 2007; Wilson et al., 2007; Herbinger et al., 2009; Clay \& de Waal, 2015; Wittig et al., 2016; Samuni et al., 2019, see supplementary material). Compared to bonobos, same-sex sexual behaviour is rare in chimpanzees, although males in the wild regularly share body contacts, including genital touches and mounting, prior to events that benefit from cooperation and group cohesiveness, such as intergroup encounters (Wittig et al., 2016; Samuni et al., 2019). Genital contacts also occur in chimpanzee conflict contexts although they are no more common than kissing, embracing, and submissive gestures (de Waal \& van Roosmalen, 1979).

Despite the prevalence of sexual behaviours in primates, fellatio whereby a partner's penis is taken into another's mouth - appears to be rare. Thus far, it has been reported between male-male dyads of laboratory-housed stumptailed macaques (Macaca arctoides; Chevalier-Skolnikoff, 1974) and during periods of crowding among male Tibetan macaques (Macaca thibetana), with the author suggesting it to play a role in greeting and tension reduction (Ogawa, 2006). Furthermore, two published reports in chim- 
panzees also documented oral-genital contact between juveniles in the context of play, however they do not specify whether fellatio was observed (Savage \& Malick, 1977; Savage-Rumbaugh \& Wilkerson, 1978). In this report, we contribute a novel observation of same-sex fellatio occurring among two sanctuary-living adult male chimpanzees occurring during a period of social tension. Arising among adults during a post-conflict period, this observation differs notably from previous unpublished observations in great apes describing the very rare occurrence of fellatio during play among captive immature chimpanzees at La Vallée des Singes, France (Heesen, personal communication) and among immature bonobos at Lola ya Bonobo Sanctuary (Clay, personal observation). By reporting on the occurrence of fellatio among adult male chimpanzees during a socially-tense period, we believe our observation can contribute to a richer understanding of the form and function of same-sex sexual behaviours in primates.

\section{Study site and methods}

A group of sanctuary-living chimpanzees was observed between March and August 2019 at Chimfunshi Wildlife Orphanage (hereafter "Chimfunshi") in the Copperbelt Province of Zambia as part of a larger research project examining post-conflict behaviours and empathic tendencies. A member of the Pan African Sanctuary Alliance (PASA), Chimfunshi houses wild-born chimpanzees rescued from the pet and bushmeat trades as well as motherreared individuals born at the sanctuary, across four stable social groups. These groups are accessible to approved observational and non-invasive research projects. Our study population (Group 2) consisted of 52 chimpanzees comprising all age- and sex-classes (see Table 1 for selected relevant IDs). The chimpanzees live in an expansive outdoor enclosure of $0.65 \mathrm{~km}^{2}$ incorporating densely vegetated miombo forest and grassland. Individuals nest independently and are fed several times per day by carers.

To assess the group-level hierarchy, we used the R package 'EloRating' to create dominance scores for 34 individuals based on dyadic agonistic interactions involving high-contact aggression, low-contact aggression, and chases (Neumann \& Kulik, 2020). Each agonism type was assigned a different optimised K-value based on intensity and likelihood of winning probabilities. Only individuals four-years and older with at least six observed dyadic agonistic interactions were included in our ELO analysis. Dominant individuals were considered those clustered in the top $25 \%$ of the group. 
Table 1.

Select IDs from Group 2 at Chimfunshi Wildlife Orphanage, Zambia.

\begin{tabular}{lcccc}
\hline ID & Sex & ${\text { Age }(\text { Years })^{\mathrm{a}}}$ & Rank $(E L O)^{\mathrm{b}}$ & Origin $^{\mathrm{c}}$ \\
\hline Daisey & $\mathrm{F}$ & 14 & 31 & $\mathrm{c}$ \\
David & $\mathrm{M}$ & 17 & 5 & $\mathrm{c}$ \\
Doug & $\mathrm{M}$ & 16 & 1 & $\mathrm{c}$ \\
Little Jack & $\mathrm{M}$ & 7 & 22 & $\mathrm{c}$ \\
Misha & $\mathrm{F}$ & 31 & 25 & $\mathrm{w}$ \\
Max & $\mathrm{d}$ & 12 & 8 & $\mathrm{c}$ \\
Maxine & $\mathrm{F}$ & 18 & 32 & $\mathrm{c}$ \\
Moyo & $\mathrm{M}$ & 11 & 6 & $\mathrm{c}$ \\
Nina & $\mathrm{F}$ & 16 & 14 & $\mathrm{c}$ \\
Tina & $\mathrm{F}$ & 4 & $\mathrm{NR}$ & $\mathrm{c}$ \\
Violet & $\mathrm{F}$ & 28 & 7 & $\mathrm{w}$ \\
Zsabu & $\mathrm{M}$ & 29 & 3 & $\mathrm{w}$ \\
\hline
\end{tabular}

a On 11 July 2019.

$\mathrm{b}$ Based on dyadic agonistic interactions including high- and low-contact aggression and chases between 16 April and 3 August 2019.

c c, captive-born; w, wild-born.

${ }^{\mathrm{d}}$ Offspring of female in previous row.

\section{Observation}

Our observation took place on 11 July 2019 at approximately 14:16 PM during a routine afternoon feeding session. It was recorded within a systematic five-minute post-conflict focal follow of Max (12-year-old male) born in Chimfunshi to Misha, a wild-born adult female. ELO dominance analyses reveal Max as the second lowest-ranked adult male (10 years and older) out of eight males in the group.

Following a chase and threat by Violet, a dominant adult female, Max expressed victim behaviour, including a bared-teeth display and screaming, and thereafter interacted with various bystanders. At $2 \mathrm{~s}$ after the conflict, Moyo, another adult male, made a bluff display towards Max, who subsequently approached Moyo submissively, who responded with a body kiss on Max's back. Approximately one minute after the original conflict with Violet, Max received a body kiss from Nina, an adult female, and was touched by Little Jack, a juvenile male. Max then mounted Tina, an infant female with thrusts (intromission was indiscernible).

Following these contacts, Max directed submissive gestures, including bared-teeth displays and crouching, towards several dominant adult male 

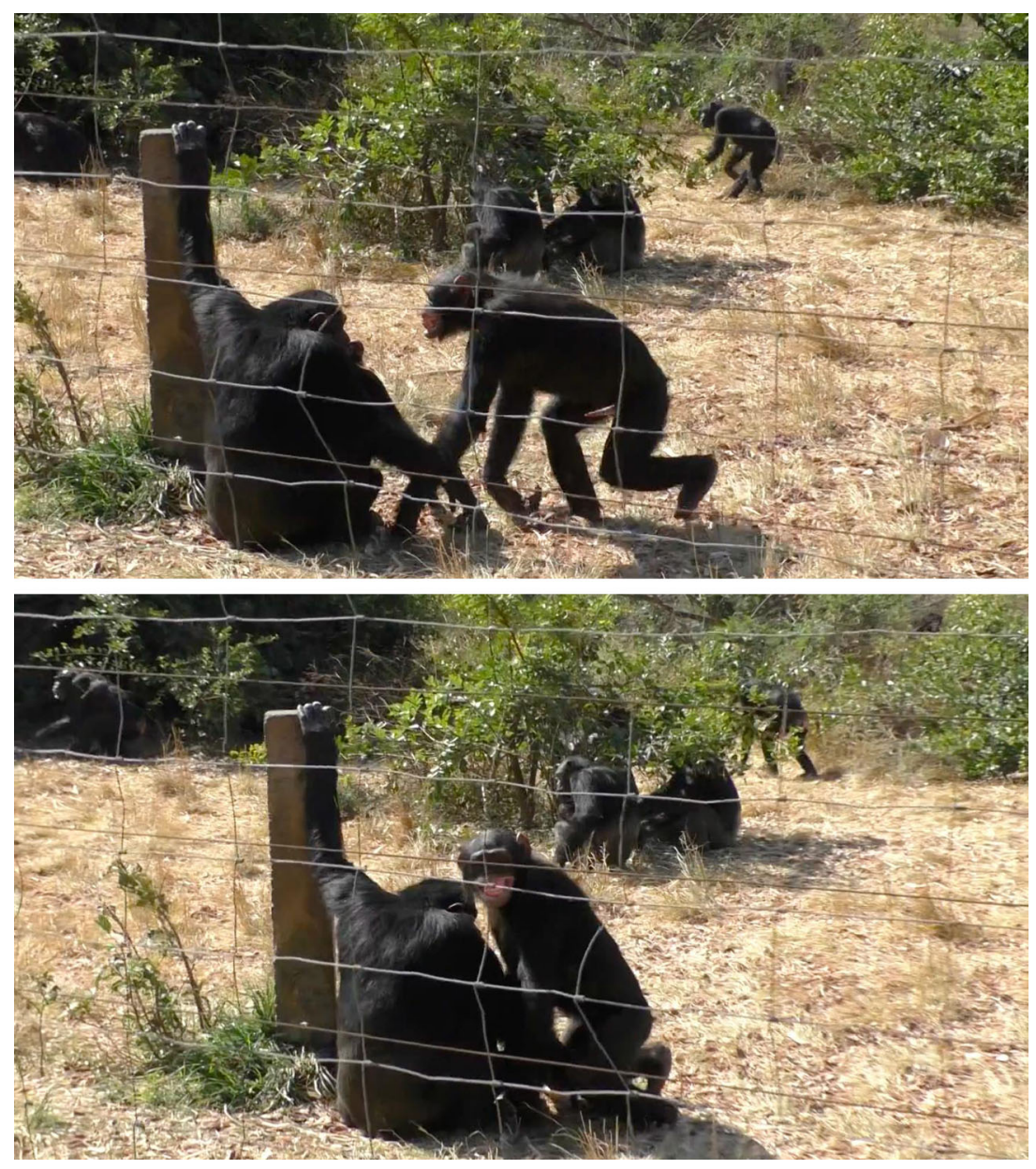

Figure 1. [01:58] Max approaching David with a bared-teeth display (top) and subsequently thrusting his erect penis towards David's lower right arm (bottom).

individuals including Doug and Zsabu. At $1 \mathrm{~min} 32 \mathrm{~s}$, Max approached David (a 17-year-old male) while presenting his penis. Max thrusted it towards David's arm (Figure 1), who appeared to touch it briefly (Figure 2) before Max bared his teeth at Zsabu, whimpered, and walked several metres away. At 2 min 7 s, David approached Max with his mouth open and gaze directed at Max's groin (Figure 3). Max placed his right knuckles on the ground, his left hand on the right side of David's face and used his hips to manoeuvre his erect penis into David's mouth (Figure 4). Max then made slight thrusts 


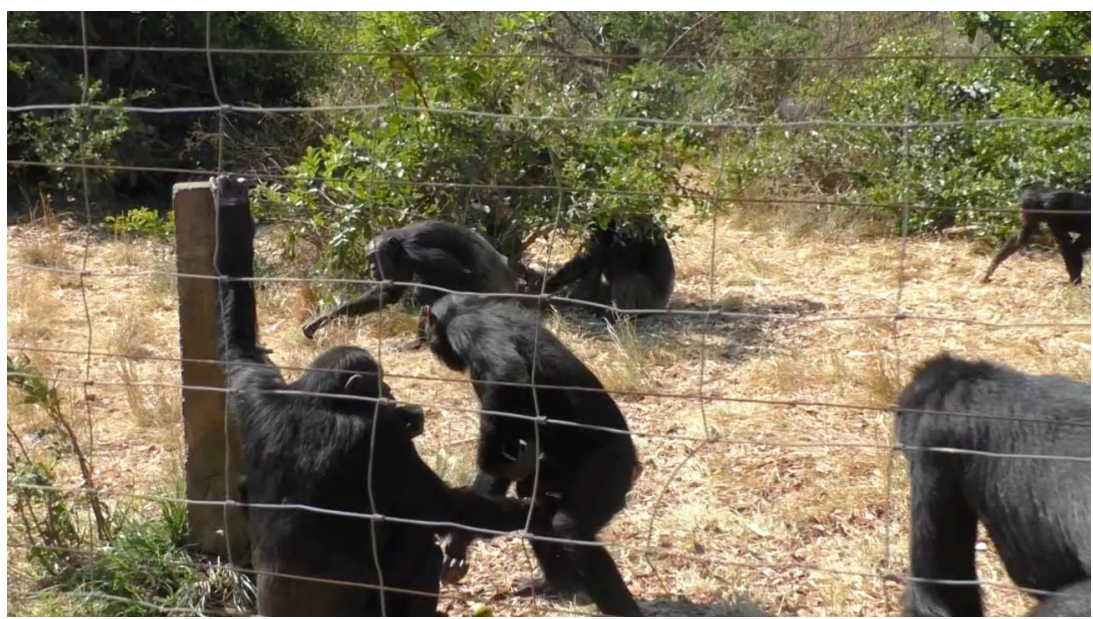

Figure 2. [01:59] David responding to Max's advance with a slight movement of his hand towards Max's penis, appearing to touch it as Max starts to walk away.

and moved his left hand to David's chin, who placed his lips around Max's penis tip (Figure 5). During the fellatio, Max bared his teeth and appeared to whimper. The fellatio lasted for approximately $3.5 \mathrm{~s}$ before Zsabu stepped towards the pair and Max withdrew. Following this encounter, Max initiated copulation with Daisey, an adult female.

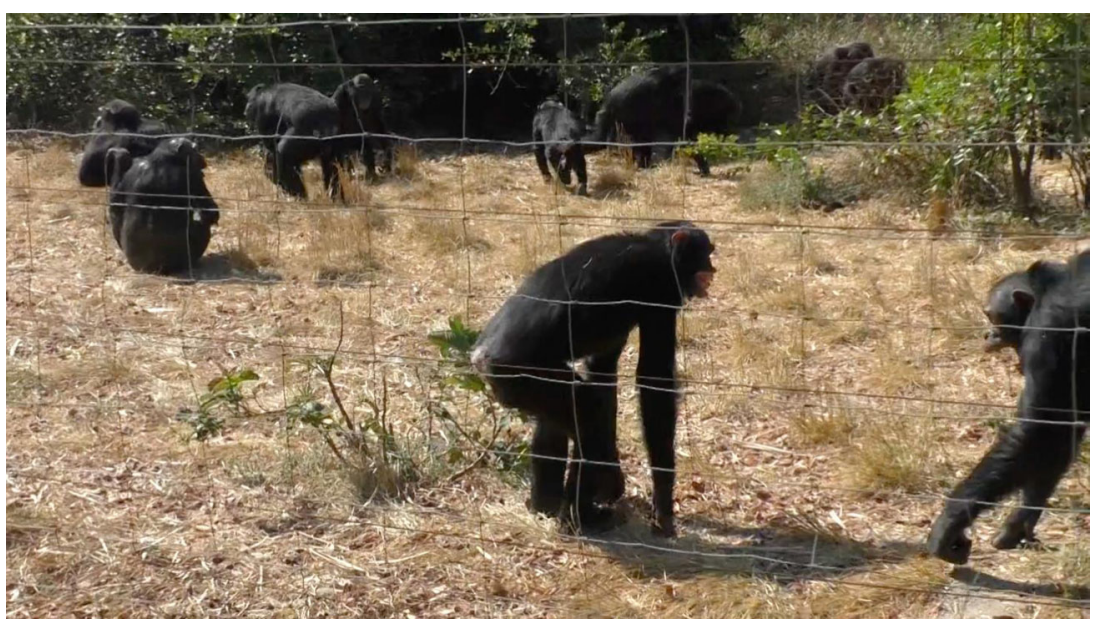

Figure 3. [02:07] Max with a bared-teeth display looking towards David who is approaching with his mouth open and teeth on display, whilst appearing to gaze towards Max's groin. 


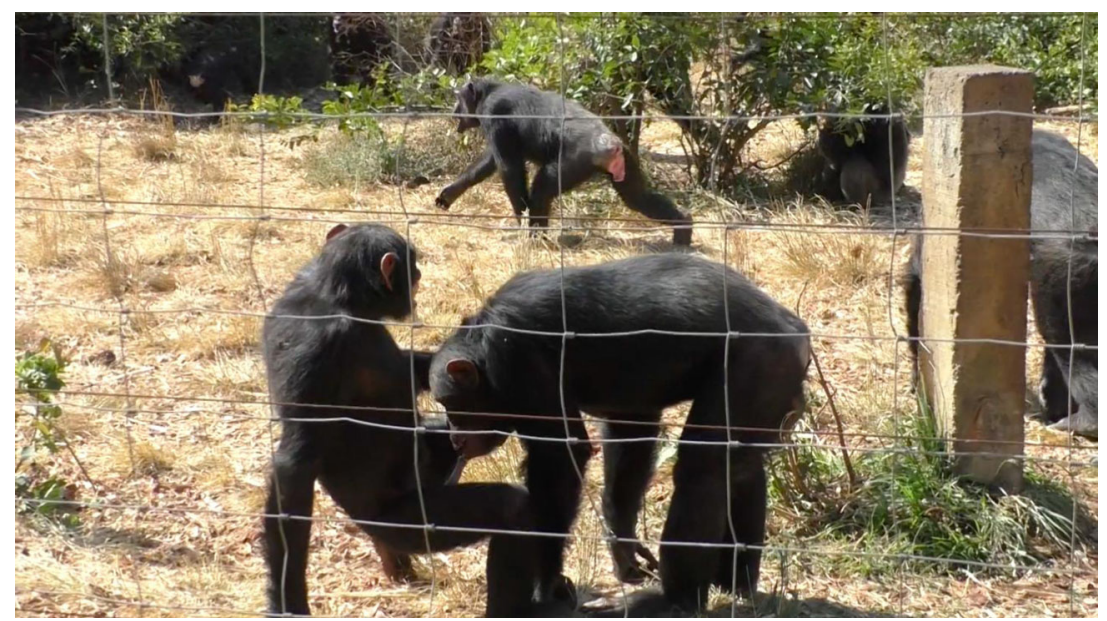

Figure 4. [02:09] Max still baring his teeth with his penis placed inside David's mouth, whilst touching the right side of David's face.

During several months prior to this interaction, Max made other unaccepted sexual invitations following dyadic conflicts where he expressed victim behaviour. On two occasions Max presented his penis towards uninvolved bystanders including on 27 April towards his adult sister Maxine, and on May 7 towards Zsabu. On these occasions, Max made a concave

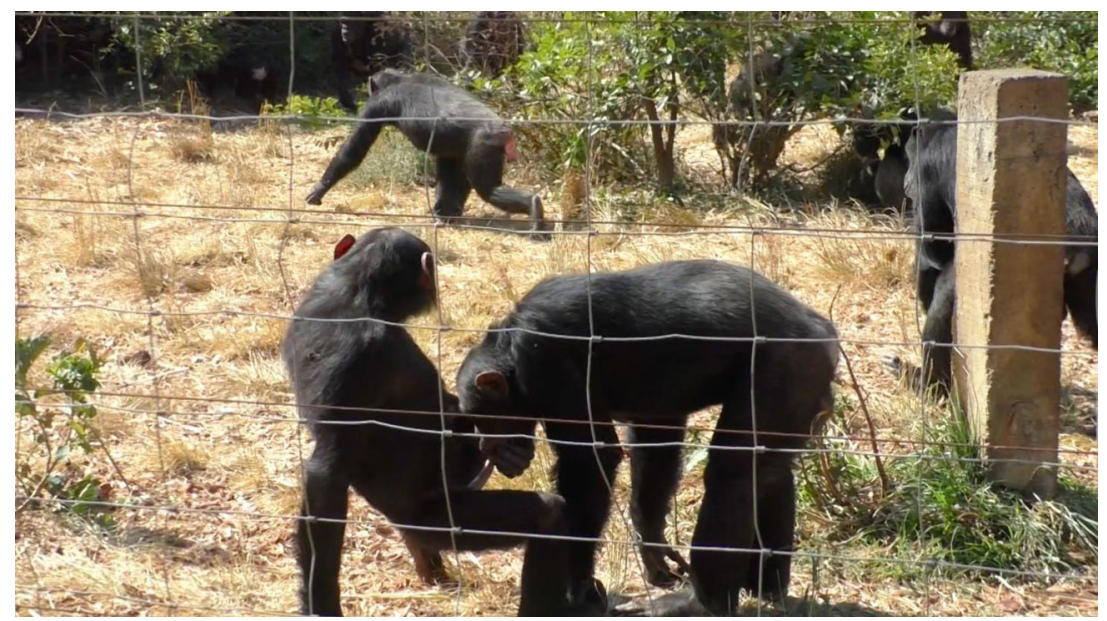

Figure 5. [02:10] Max has moved his hand to David's chin and makes gentle thrusts with his hips, moving his penis slightly in and out of David's mouth. 
back and thrusted his erect penis towards the recipient with one or both hands placed on the ground behind his feet. On July 6, the only observed dyadic agonistic interaction between Max and David occurred. David chased Max who fled and responded with a bared-teeth display and presented his rump towards David who did not appear to engage. On two occasions during high-tension feeding periods following the observed fellatio (13 July and 19 July), Max ventro-dorsally embraced David with pelvic thrusts. We did not observe ejaculation during any of these events. All of these interactions are video-recorded and can be seen at 10.6084/m9.figshare.13325171. Social affiliation scan data from the full observation period revealed no recording of Max and David gathering in close proximity $(<1$ metre) or engaging in affiliative behaviours, including grooming or play.

\section{Discussion}

In this report, we described an instance of same-sex fellatio occurring between two sanctuary-living adult male chimpanzees. Although fellatio has been observed in other mammals (Ogawa, 2006; Tan et al., 2009; Sergiel et al., 2014; Sugita, 2016), including captive/semi-captive bonobos (de Waal, 1988; Clay, personal observation) and chimpanzees (Heesen, personal communication) it has not yet been reported among adult chimpanzees during social tension, with two previous reports noting the occurrence of oralgenital contact among play in immature chimpanzees (Savage \& Malick, 1977; Savage-Rumbaugh \& Wilkerson, 1978). Moreover, while bonobos habitually engage in diverse same-sex sexual behaviour, fellatio appears to be rare and constrained to play contexts involving immatures (de Waal, 1988; Clay, personal observation). To our knowledge, fellatio has not been reported to occur in post-conflict affiliative interactions among either chimpanzees or bonobos. This observation thus provides relevant insights into the potential diversity of its functions in our closest living relatives.

Homosexual interactions, such as those reported here between Max and David, have been hypothesised to reinforce same-sex alliances and increase the propensity to support and cooperate (Kirkpatrick, 2000; Moscovice et al., 2019). Creating such connections may facilitate alliance formation and provide greater opportunities for future cooperation and support during agonism between non-kin. As a low-ranking chimpanzee, it is possible that Max's request for fellatio in this case may have been driven by a motivation 
to associate with potential coalition partners, with genital contacts having been shown to facilitate coalitionary support in bonobos, the close relative of chimpanzees (Moscovice et al., 2019). As our closest phylogenetic relatives, studying genital contacts in chimpanzees, including fellatio, in specific contexts may provide clarity on the evolutionary origins of same-sex sexual behaviour within and between the sexes.

Our observation may also reflect fellatio serving a reassurance and alliance testing function by a subordinate male, akin to genital touches and mounting commonly observed between wild male chimpanzees during socially tense periods such as before boundary patrols and intergroup encounters (Wittig et al., 2016; Samuni et al., 2019). It has been proposed that engaging in intimate, risky behaviour such as fellatio can be used to test social relationships and tolerance (Kirkpatrick, 2000).

Chimpanzees are behaviourally innovative and spontaneously develop unique behaviour that may culturally transmit, even if such actions appear to lack adaptive benefits (van Leeuwen et al., 2014). As chimpanzees are not known to habitually engage in same-sex sexual interactions, Max's repeated attempts to initiate genital contact for reassurance may also represent a behavioural innovation. Observing Max's group in varying contexts would be necessary to detect whether he possesses a more general tendency for same-sex sexual interactions. Further, longitudinal investigations may show that idiosyncratic sexual behaviour, such as fellatio, is culturally transmitted over time.

Although the functions of fellatio remain to be explored in great apes, its initiation by a lower ranking to a dominant male in a socially tense context makes a novel contribution to the literature. Given the apparent overlaps between this behaviour and genital contacts occurring among the close sister species, the bonobos, systematically comparing sexual behaviours during periods of social tension between Pan would provide greater clarity on how sexual behaviours may be adapted and deployed to fortify and repair social relationships.

\section{Acknowledgements}

We thank the staff members of Chimfunshi Wildlife Orphanage, the Chimfunshi Research Advisory Board, Edwin van Leeuwen, Zoë Goldsborough, Raphaela Heesen, and Frans de Waal. This project is part of a larger research 
project funded by the Templeton World Charity Foundation's Diverse Intelligences Initiative (Grant No. TWCF0309), and was approved by the Animal Welfare Ethical Review Board of Durham University. We thank three anonymous reviewers for their helpful comments on earlier drafts of the manuscript.

\section{References}

Bailey, N.W. \& Zuk, M. (2009). Same-sex sexual behavior and evolution. — Trends Ecol. Evol. 24: 439-446.

Chevalier-Skolnikoff, S. (1974). Male-female, female-female, and male-male sexual behavior in the stumptail monkey, with special attention to the female orgasm. - Arch. Sex. Behav. 3: 95-116.

Clay, Z. \& de Waal, F.B.M. (2013). Bonobos respond to distress in others: consolation across the age spectrum. - PLoS ONE 8: e55206.

Clay, Z. \& de Waal, F.B.M. (2015). Sex and strife: post-conflict sexual contacts in bonobos. — Behaviour 152: 313-334.

de Waal, F.B.M. (1988). The communicative repertoire of captive bonobos (Pan paniscus), compared to that of chimpanzees. - Behaviour 106: 183-251.

de Waal, F.B.M. (1990). Sociosexual behavior used for tension regulation in all age and sex combinations among bonobos. - In: Pedophilia: biosocial dimensions (Fireman, J.R., ed.). Springer, New York, NY, p. 378-393.

de Waal, F.B.M. \& van Roosmalen, A. (1979). Reconciliation and consolation among chimpanzees. - Behav. Ecol. Sociobiol. 5: 55-66.

Goodall, J. (1989). Glossary of chimpanzee behaviors. - Jane Goodall Institute, Tucson, AZ.

Hanby, J.P. (1976). Sociosexual development in primates. - In: Perspectives in ethology, Vol. II (Bateson, P.P.G. \& Klopfer, P.H., eds). Plenum Press, New York, NY, p. 1-6.

Herbinger, I., Papworth, S., Boesch, C. \& Zuberbühler, K. (2009). Vocal, gestural and locomotor responses of wild chimpanzees to familiar and unfamiliar intruders: a playback study. - Anim. Behav. 78: 1389-1396.

Kirkpatrick, R.C. (2000). The evolution of human homosexual behavior. - Curr. Anthropol. 41: 385-413.

Moscovice, L.R., Surbeck, M., Fruth, B., Hohmann, G., Jaeggi, A.V. \& Deschner, T. (2019). The cooperative sex: sexual interactions among female bonobos are linked to increases in oxytocin, proximity and coalitions. - Horm. Behav. 116: 104581.

Neumann, C. \& Kulik, L. (2020). EloRating - a brief tutorial. — CRAN 2020-03-12 (v. $0.46 .11)$.

Ogawa, H. (2006). Wily monkeys: social intelligence of Tibetan macaques. — Kyoto University Press, Kyoto.

Paoli, T., Tacconi, G., Tarli, S.M.B. \& Palagi, E. (2007). Influence of feeding and short-term crowding on the sexual repertoire of captive bonobos (Pan paniscus). - Ann. Zool. Fenn. 44: 81-88. 
Samuni, L., Preis, A., Deschner, T., Wittig, R.M. \& Crockford, C. (2019). Cortisol and oxytocin show independent activity during chimpanzee intergroup conflict. - Psychoneuroendocrinology 104: 165-173.

Savage, E.S. \& Malick, C. (1977). Play and socio-sexual behaviour in a captive chimpanzee (Pan troglodytes) group. - Behaviour 60: 179-194.

Savage-Rumbaugh, E.S. \& Wilkerson, B.J. (1978). Socio-sexual behavior in Pan paniscus and Pan troglodytes: a comparative study. - J. Hum. Evol. 7: 327-344.

Sergiel, A., Maślak, R., Zedrosser, A., Paśko, Ł., Garshelis, D.L., Reljić, S. \& Huber, D. (2014). Fellatio in captive brown bears: evidence of long-term effects of suckling deprivation?. - Zoo. Biol. 33: 349-352.

Sommer, V. \& Vasey, P.L. (2006). Homosexual behaviour in animals: an evolutionary perspective. - Cambridge University Press.

Sugita, N. (2016). Homosexual fellatio: erect penis licking between male Bonin flying foxes Pteropus pselaphon. - PLoS ONE 11: e0166024.

Tan, M., Jones, G., Zhu, G., Ye, J., Hong, T., Zhou, S., Zhang, S. \& Zhang, L. (2009). Fellatio by fruit bats prolongs copulation time. - PLoS ONE 4: e7595.

van Leeuwen, E.J.C., Cronin, K.A. \& Haun, D.B.M. (2014). A group-specific arbitrary tradition in chimpanzees (Pan troglodytes). - Anim. Cogn. 17: 1421-1425.

Vasey, P.L. (1996). Interventions and alliance formation between female Japanese macaques, Macaca fuscata, during homosexual consortships. — Anim. Behav. 52: 539-551.

Vasey, P.L. (2006). The pursuit of pleasure: homosexual behaviour, sexual reward and evolutionary history in Japanese macaques. - In: Homosexual behaviour in animals: an evolutionary perspective (Sommer, V. \& Vasey, P.L., eds). Cambridge University Press, Cambridge, p. 191-219.

Wilson, M.L., Hauser, M.D. \& Wrangham, R.W. (2007). Chimpanzees (Pan troglodytes) modify grouping and vocal behaviour in response to location-specific risk. - Behaviour 144: 1621-1653.

Wittig, R.M., Crockford, C., Weltring, A., Langergraber, K.E., Deschner, T. \& Zuberbühler, K. (2016). Social support reduces stress hormone levels in wild chimpanzees across stressful events and everyday affiliations. - Nature Commun. 7: 1-8. 